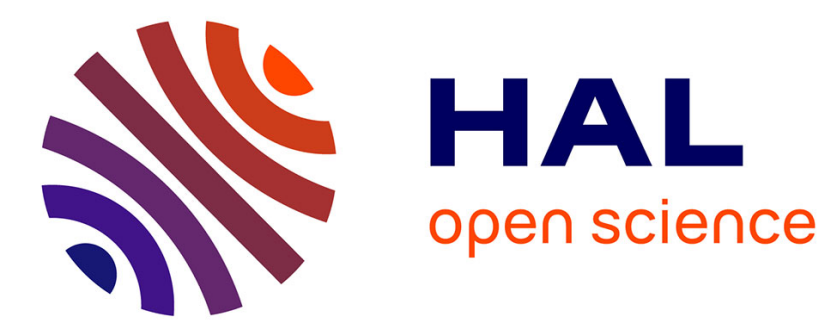

\title{
Vortical control of forced two-dimensional turbulence
}

\author{
Jerome Fontane, David Dritschel, Richard Scott
}

\section{To cite this version:}

Jerome Fontane, David Dritschel, Richard Scott. Vortical control of forced two-dimensional turbulence. Physics of Fluids, 2013, vol. 25, 10.1063/1.4774336 . hal-00786342

\section{HAL Id: hal-00786342 \\ https://hal.science/hal-00786342}

Submitted on 8 Feb 2013

HAL is a multi-disciplinary open access archive for the deposit and dissemination of scientific research documents, whether they are published or not. The documents may come from teaching and research institutions in France or abroad, or from public or private research centers.
L'archive ouverte pluridisciplinaire HAL, est destinée au dépôt et à la diffusion de documents scientifiques de niveau recherche, publiés ou non, émanant des établissements d'enseignement et de recherche français ou étrangers, des laboratoires publics ou privés. 


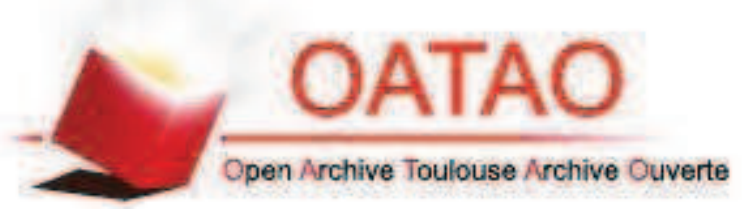

\section{Open Archive Toulouse Archive Ouverte (OATAO)}

OATAO is an open access repository that collects the work of Toulouse researchers and makes it freely available over the web where possible.

This is an author-deposited version published in: http://oatao.univ-toulouse.fr/ Eprints ID: 8397

To link to this article: DOI: $10.1063 / 1.4774336$

URL: http://ink.aip.org/link/doi/10.1063/1.4774336

To cite this version: Fontane, Jerome and Dritschel, David and Scott, Richard Vortical control of forced two-dimensional turbulence. (2013) Physics of Fluids, vol. 25 ( $\mathrm{n}^{\circ}$ 1). ISSN 1089-7666

Any correspondence concerning this service should be sent to the repository administrator: staff-oatao@inp-toulouse.fr 


\title{
Vortical control of forced two-dimensional turbulence
}

\author{
Jérôme Fontane, ${ }^{1,2}$ David G. Dritschel, ${ }^{1}$ and Richard K. Scott ${ }^{1}$ \\ ${ }^{1)}$ School of Mathematics and Statistics, University of St Andrews, St Andrews KY16 9SS, \\ United Kingdom \\ ${ }^{2)}$ Université de Toulouse, Institut Supérieur de l'Aéronautique et de l'Espace, \\ 10 avenue Édouard Belin - BP 54032 - 31055 Toulouse Cedex 4, France
}

(Dated: 4 January 2013)

A new numerical technique for the simulation of forced two-dimensional turbulence ${ }^{1}$ is used to examine the validity of Kraichnan-Batchelor scaling laws at higher Reynolds number than previously accessible with classical pseudo-spectral methods, making use of large simulation ensembles to allow a detailed consideration of the inverse cascade in a quasi-steady state. Our results support the recent finding of Scott ${ }^{2}$, namely that when a direct enstrophy cascading range is well-represented numerically, a steeper energy spectrum proportional to $k^{-2}$ is obtained in place of the classical $k^{-5 / 3}$ prediction. It is further shown that this steep spectrum is associated with a faster growth of energy at large scales, scaling like $t^{-1}$ rather than Kraichnan's prediction of $t^{-3 / 2}$. The deviation from Kraichnan's theory is related to the emergence of a population of vortices that dominate the distribution of energy across scales, and whose number density and vorticity distribution with respect to vortex area are related to the shape of the enstrophy spectrum. An analytical model is proposed which closely matches the numerical spectra between the large scales and the forcing scale.

\section{INTRODUCTION}

Density stratification and planetary rotation place strong constraints on geophysical flows in a wide range of situations, from the Jovian atmosphere to the Earth's oceans. These flows can be represented to a good approximation by layer-wise two-dimensional flow with varying degrees of vertical coherence; high Reynolds number, two-dimensional turbulence is commonly considered as one of the simplest models to assess qualitative aspects of their dynamics. Due to the existence of two invariants, energy and enstrophy, this model exhibits the most remarkable feature of two-dimensional flows, namely a dual cascade in which energy is transferred preferentially to large scales, while enstrophy is transferred preferentially to small scales. Both cascades are associated with inertial ranges characterised by a power-law distribution of the relevant quantity across scales. Assuming constant energy and enstrophy fluxes, $\epsilon$ and $\eta$, respectively, and assuming locality of interactions between different scales, Kraichnan ${ }^{3}$ showed in his pioneering theoretical work that the energy is distributed across wavenumbers $k$ as $E(k)=C \epsilon^{2 / 3} k^{-5 / 3}$ for $k<k_{f}$ and $E(k)=C \eta^{2 / 3} k^{-3}$ for $k>k_{f}$, where $k_{f}$ is the wavenumber at which energy is input into the system. The form of the enstrophy inertial range in the freely-decaying case was also found shortly thereafter by Batchelor ${ }^{4}$ and is now commonly referred to as the Batchelor spectrum. Kraichnan ${ }^{5}$ later introduced a weak logarithmic correction to take into account the effect of non-locality in the enstrophy inertial range, suggesting a modified spectrum of the form $E(k)=C \eta^{2 / 3} k^{-3} \log \left(k / k_{f}\right)^{-1 / 3}$.

Since then, a substantial number of studies have been carried out to validate the Kraichnan-Batchelor theory by means of numerical simulations ${ }^{6-14}$ and physical experiments ${ }^{15-18}$. While most of them have confirmed the Kraichnan-Batchelor theory, three numerical works found a deviation from the $k^{-5 / 3}$ spectrum in the inverse energy cascade range where a steeper slope was measured: Borue ${ }^{11}$ observed spectra as steep as $k^{-3}$ while Smith \& Yakhot $^{9}$ and Danilov \& Gurarie $^{12}$ observed spectra of the form $k^{-n}$ with $2<n<2.3$ and $2.2<n<2.5$ respectively - even in special cases with no large-scale energy dissipation. All these authors attributed these deviations to the emergence of a population of coherent vortices. As concluded by Danilov \& Gurarie $^{12}$, the universal $k^{-5 / 3}$ seems 'exceptional and unstable'. But all of these studies ${ }^{6-18}$ suffer from at least one of the two following issues that have a strong influence on both cascades, which put in question the reliability of their results.

First, most studies have focussed on only one of the inertial ranges due to numerical or experimental constraints. In these studies, energy is typically injected close to the largest (or smallest) resolved scale in order to produce a direct (or inverse) cascade over a range of scales as wide as possible. More recently, however, it has become possible to assess the effect of the representation of one cascade on the dynamics of the other. This was first considered by Tran \& Bowman ${ }^{19}$, who showed that even in the absence of a direct enstrophy cascade, i.e. for energy spectra steeper than $k^{-5}$ in the enstrophy inertial range, the spectrum in the energy inertial range is not affected, only the strength of the cascade is altered. This underscores the robustness of the energy inverse cascade which was commonly observed in previous works. On the other hand, Scott ${ }^{2}$ demonstrated that the slope of the inverse cascade may not be robust to increases in the width of the enstrophy cascading range. Measuring the width of the enstrophy range by the ratio $k_{\max } / k_{f}$ where $k_{\max }$ is the maximal resolved wavenumber, for regular viscosity it was shown that when this ratio exceeds a value of around $k_{\max } / k_{f}=16$ (corresponding to the onset of the direct cascade) the inverse cascade steepens from $k^{-5 / 3}$ to $k^{-2}$. When hyperviscosity is used in place of viscos- 
ity, a similar steepening occurs, but for a lower value of $k_{\max } / k_{f} \sim 8$. The ratio must be increased still further, to a value of around $k_{\max } / k_{f} \sim 64$, before the inverse cascade approaches full strength, in the sense that almost all of the energy input is transferred to larger scales. The steepening for large $k_{\max } / k_{f}$ was again attributed to the emergence of a population of coherent vortices that dominates the energy distribution in the inverse cascade. A recent paper by Vallgren ${ }^{20}$ using a pseudospectral model at extremely high resolution concluded also that the inverse cascade is steeper than $k^{-5 / 3}$ at large $k_{\max } / k_{f}$, supporting the results of Scott, and identified the importance of spectrally nonlocal energy transfers associated with coherent vortices. On the other hand, it was found that the spectrum associated with the incoherent background of vorticity filaments does obey the Kraichnan-Batchelor scaling.

The second issue that requires careful attention is the implementation of large-scale friction or hypo-diffusion, often used in previous studies, to remove energy at large scales. This is typically done to allow a statistical steady state to be reached, in which energy input is balanced by energy removal, and to prevent the build up of energy in the lowest modes, a phenomenon sometimes referred to as 'condensation', ${ }^{\prime 9}$. The use of such energy dissipation, however, typically distorts the inertial range, either through large-scale bottleneck effects in the case of hypodiffusion, or by destroying the assumption of constant energy flux in the case of friction ${ }^{12,21}$. The last point is important when considering the recent numerical simulations of Boffetta ${ }^{22}$ and Boffetta \& Musacchio ${ }^{23}$, who obtained a $k^{-5 / 3}$ spectrum but in the presence of linear friction $^{35}$. In the paper of Vallgren ${ }^{20}$, the effect of linear friction was also considered and found to lead to slopes that varied between Kraichnan's $-5 / 3$ prediction and a significantly steeper value of -2.4 .

All these previous studies that found deviations from the Kraichnan-Batchelor theory were either using a lowwavenumber energy dissipation ${ }^{11,20,23}$ or, otherwise, were forcing the flow close to the dissipation scale: the ratio $k_{\max } / k_{f}$ varied between 3 and 5 for Borue ${ }^{11}$, between 4 and 5 for Smith \& Yakhot ${ }^{9}$ and between 1 and 4 for Danilov \& Gurarie ${ }^{12}$. So none of these studies were designed to avoid simultaneously these two problems and this, we believe, is why there is such a variability in the measured spectral slope of the inverse cascade. The purpose of the present work is to provide (i) additional validation of a steeper inverse cascade than theorized by Kraichnan $^{3}$ and found in many previous numerical studies, and (ii) detailed analysis of the vortex population which emerges and modifies this cascade. We avoid the use of frictional or other large-scale damping, and ensure adequate resolution of the enstrophy cascade range by taking $k_{\max } / k_{f}=64$.

To this end, we examine numerical simulations which are original in two respects. First, we exploit the latest generation of powerful algorithms specially designed for geophysical flows ${ }^{1}$. The Combined Lagrangian Ad- vection Method (CLAM) is a hybrid method that combines the efficiency of Lagrangian contour dynamics with the energy conservation properties of the pseudo-spectral method. Its use allows the simulation of much higher Reynolds number than is possible with traditional (e.g. purely pseudo-spectral) methods. In contrast to pseudospectral methods, where regular viscous or hyperviscous schemes must be added for numerical stability, no explicit diffusion term is needed here. Weak dissipation arises from the use of contour surgery ${ }^{25,26}$, which is used only to control the complexity of vorticity contours. Importantly, surgery takes place at a scale well below the grid size and has no diffusive effect whatsoever on steep vorticity gradients. In the freely decaying case, these hybrid algorithms were proved to be less diffusive, more accurate and considerably more efficient than pseudo-spectral methods ${ }^{27,28}$. Second, because no large-scale damping is used, statistical convergence is obtained by means of ensemble-averages of many simulations, which to our knowledge has never been done previously in forced 2D turbulence. Our simulations may be considered quasistationary in the sense that the energy levels at a given scale in the inverse cascade remain constant while the length of the inverse cascade and the total energy of the flow increases. Our approach is therefore consistent with Kraichnan's original theory, in which energy is assumed to cascade, undissipated, to ever larger scales. This implies that the flow is continuously evolving, and hence analyses based on time averaging are generally inappropriate.

The remainder of the paper is organised as follows. In section two, we describe the numerical simulations and give a short overview of the CLAM algorithm and its advantages over other methods in current use. In section three we present the main results, describing the general evolution of the flow and the evolution of the energy distribution in spectral space; this is followed by an analysis of the vorticity field in physical space, showing that the deviation from the Kraichnan-Batchelor theory is associated with the emergence of a population of coherent vortices distributed across a range of length scales. We conclude in section four with a short summary.

\section{NUMERICAL SIMULATIONS}

Using the streamfunction $\psi$ and vorticity $\omega$, the twodimensional Euler equations governing the motion of an incompressible fluid submitted to external forcing $f$ can be written as

$$
\partial_{t} \omega+J(\psi, \omega)=f,
$$

where $\omega$ and $\psi$ are related by Poisson's equation $\omega=$ $\boldsymbol{\Delta} \psi$ and the two-dimensional Jacobian is $J(\psi, \omega)=$ $\partial_{x} \psi \partial_{y} \omega-\partial_{y} \psi \partial_{x} \omega$. The velocity field $\boldsymbol{u}$ is given by $\boldsymbol{u}=\left(-\partial_{y} \psi, \partial_{x} \psi\right)$. The external forcing $f$ is chosen as a $\delta$-correlated in time enstrophy input localised at wavenumber $k_{f}$, i.e. its correlation function satisfies 


\begin{tabular}{lcc}
\hline \hline Set & $\mathrm{A}$ & $\mathrm{B}$ \\
Inversion grid resolution & $N=256$ & $N=512$ \\
Contouring grid resolution & $N^{\prime}=4096$ & $N^{\prime}=8192$ \\
Forcing wavenumber & $k_{f}=32$ & $k_{f}=64$ \\
Maximal resolved wavenumber & $k_{\max }=2048$ & $k_{\max }=4096$ \\
Half band width of the forcing & $\Delta k=2$ & $\Delta k=4$ \\
Effective Reynolds number & $9.5 \times 10^{6}$ & $3.8 \times 10^{7}$ \\
Average duration of the simulations based on $\omega_{\max }$ & $297 T_{\text {eddy }}$ & $346 T_{e d d y}$ \\
Average duration of the simulations based on $\omega_{r m s}$ & $28.6 T_{r m s}$ & $28.9 T_{r m s}$ \\
\hline \hline
\end{tabular}

TABLE I. Numerical parameters used of the two sets of 25 simulations performed. The duration of the simulations is computed in terms of both the time integrated maximum vorticity and the time integrated rms vorticity.

$\left\langle f_{k}(t) f_{k}^{*}\left(t^{\prime}\right)\right\rangle=F(k) \delta\left(t-t^{\prime}\right)$ with spectrum $F(k)=$ $\eta /(2 \Delta k)$ for $\left|k-k_{f}\right| \leq \Delta k$ and $F(k)=0$ otherwise. Here the enstrophy input rate (per unit area) is chosen to be $\eta=c(2 \pi)^{2}$ with $c=0.2$, without loss of generality. The flow is doubly-periodic in a domain of dimensions $2 \pi \times 2 \pi$ and starts from a state of rest.

The simulations are performed using the Combined Lagrangian Advection Method (CLAM), a new hybrid numerical method for simulating layerwise-two-dimensional geophysical flows combining spectral methods, contour advection and vortex-in-cell methods ${ }^{1}$. The spectral method is used to represent large scales, Lagrangian contours are used to represent intermediate and small scales, and Lagrangian particles are used to represent forcing and dissipation, i.e. the narrow-band forcing $f$ in the present case. Developed from the CASL ${ }^{29,30}$ and HyperCASL $^{28,31}$ algorithms, CLAM takes advantage of each of the methods and achieves a substantially greater efficiency and accuracy than is possible when using any one element on its own. No dissipation scheme is introduced either at large or at small scales. The flow evolution is nearly inviscid with exceptionally weak numerical dissipation arising from surgery ${ }^{25,26}$ or from contour and point vortex regularisation ${ }^{28}$. In particular, contour surgery has no diffusive effect on steep gradients of vorticity.

To obtain a statistical steady state and allow temporal averaging, most previous numerical studies have used a form of damping to remove energy at large scales. As already mentioned, however, such damping may be inconsistent with Kraichnan's theory and can significantly alter the nature of the inverse cascade ${ }^{12,21}$. To avoid this problem, statistical convergence is instead obtained by averaging over an ensemble of simulations. Here, two sets of 25 simulations are performed at two different resolutions: $N=256$ for set A and $N=512$ for set B where $N$ is the number of grid points of the principal 'inversion' grid where the velocity field is computed by inversion of the vorticity field. The use of an underlying ultra-fine 'contouring' grid together with the advection of contours enables one to resolve scales far below those associated with the inversion grid. The effective resolution $N^{\prime}$ associated with the contours is sixteen times finer than the main one $N$, giving values respectively of $N^{\prime}=4096$ and
$N^{\prime}=8192^{28}$. In set A, the effective Reynolds number ${ }^{27}$ is $9.5 \times 10^{6}$, while in set B it is $3.8 \times 10^{7}$. All simulations last 50 units of time which corresponds to nearly 300 eddy turnaround times $T_{e d d y}$ based on the time integrated maximum vorticity, or $30 T_{r m s}$ based on the time integrated rms vorticity. At that stage the energy has already reached the largest scales of the flow as shown in the next section.

The forcing wavenumber is chosen in order to maximise the extent of the inverse energy cascade while preserving an accurate representation of the direct enstrophy cascade. According to $\mathrm{Scott}^{2}$ a strong inverse cascade is approached for $k_{\max } / k_{f} \approx 64$, where $k_{\max }$ is the maximum resolved wavenumber and $k_{f}$ is the forcing wavenumber. This gives $k_{f}=32$ for set $\mathrm{A}$ and $k_{f}=64$ for set B. For comparison, Boffetta ${ }^{22}$ used a ratio of 55 for the highest resolution and Vallgren ${ }^{20}$ used a ratio of 25 . All the numerical parameters are summarised in Table I. Unless stated, all data presented in the paper are produced from averaging over the 25 simulations of each set. We verified that 25 runs are enough to ensure statistical convergence up to the second statistical moments (variance) for both energy and enstrophy.

\section{RESULTS}

\section{A. General flow evolution}

Figure 1 presents several stages in the evolution of the flow, at $t=1,5,10$ and 50 , for one representative simulation in set B. In the earliest stage shown, the direct effect of the forcing is evident in the characteristic blurry honeycomb pattern of the narrow-band forcing; the flow has not yet had time to evolve significantly. By the next stage, one can already see vortices emerging and growing in a background composed of debris and filaments of vorticity. The later stages are broadly similar, albeit with more fine-scale structure and marginally more organised large-scale vortices (which contain more than $70 \%$ of the total enstrophy by $t=50$ ) as the energy grows by cascading slowly to large scales.

The temporal evolution of both energy $\mathcal{E}$ and enstrophy $\mathcal{Z}$ (ensemble averaged) is plotted in figure 2 for 

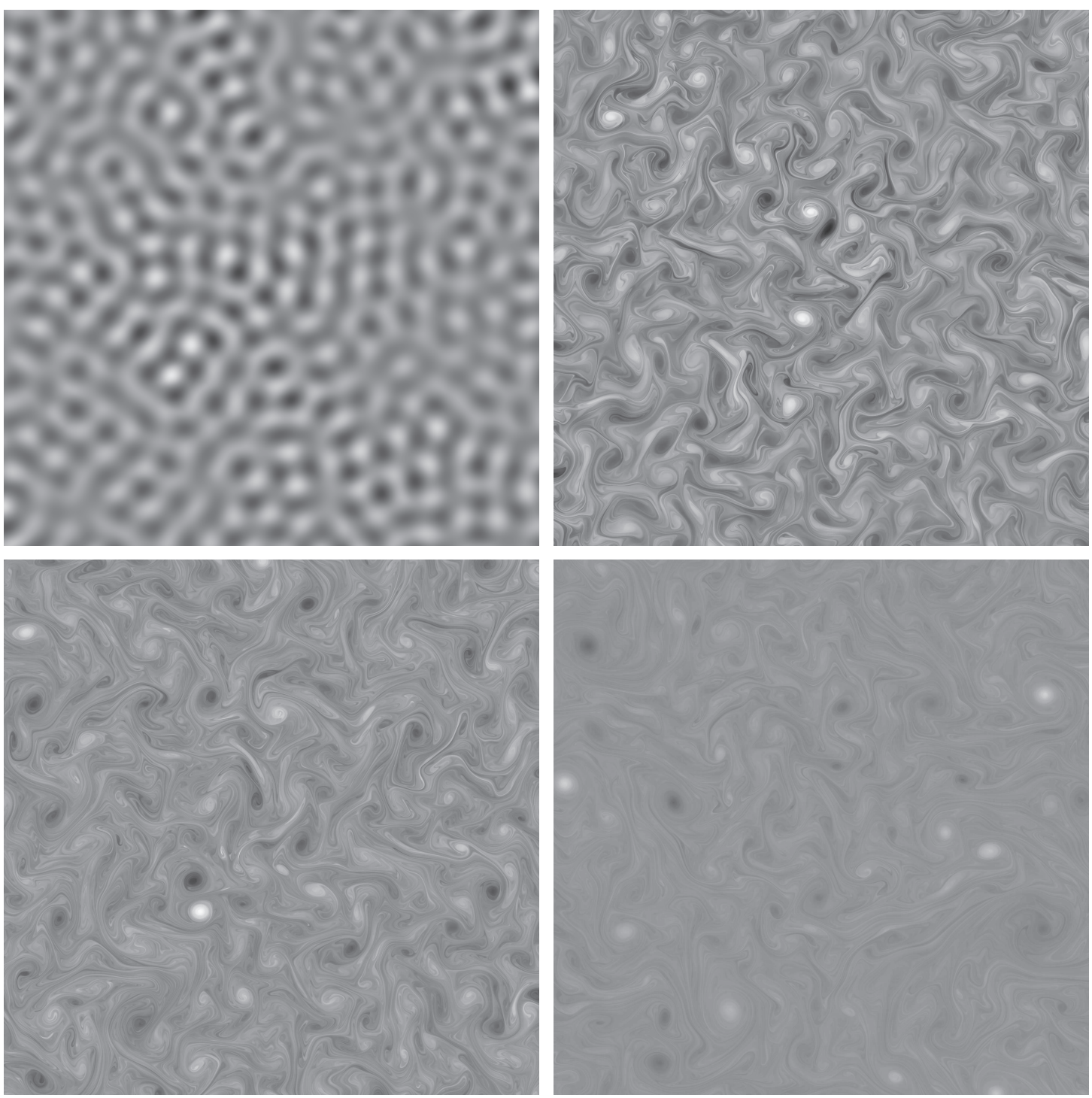

FIG. 1. The vorticity field $\omega$ at times $t=1,5,10$ and 50 from left to right and top to bottom. A linear grey scale is used with white being the highest level of vorticity value and black being the lowest. The fields are taken from one simulation in set $\mathrm{B}$ and only a sixteenth of the domain is represented.

the two sets of simulations together with the theoretical growth expected for an inviscid fluid, i.e. an enstrophy growth rate of $\eta=7.8957$ which corresponds to an energy growth rate of $\varepsilon=0.0077409$. For the duration of the simulation, energy grows close to the imposed rate while being slightly dissipated over the times considered. At $t=50$, the value of the energy is only $5.4 \%$ smaller than the theoretical value for set $\mathrm{A}$ and $5.8 \%$ smaller for set B. On the other hand, enstrophy grows at the imposed rate up to $t=4$ at which time the direct cascade reaches the smallest resolved scales and vorticity begins to be dissipated through surgery. Thereafter, a smaller linear growth in enstrophy continues until late times due to a small but non-zero fraction of the input enstrophy cascading to large scales (consistent with the energy cas- cading to large scales as discussed next).

\section{B. Spectral evolution}

Figure 3 shows the evolution of the enstrophy spectrum for both sets. Curves are plotted at times 1, 5, 10 and then every 10 time units up to the final time of the simulations. After $t=5$, which is shortly after the time when the enstrophy reaches the smallest scales of the flow and starts being dissipated, the spectra converge to a fixed form and do not evolve further in time except at small wavenumbers where the inverse cascade continues to build energy at large scales. The part of the spectrum lying in the enstrophy cascade range, i.e. 

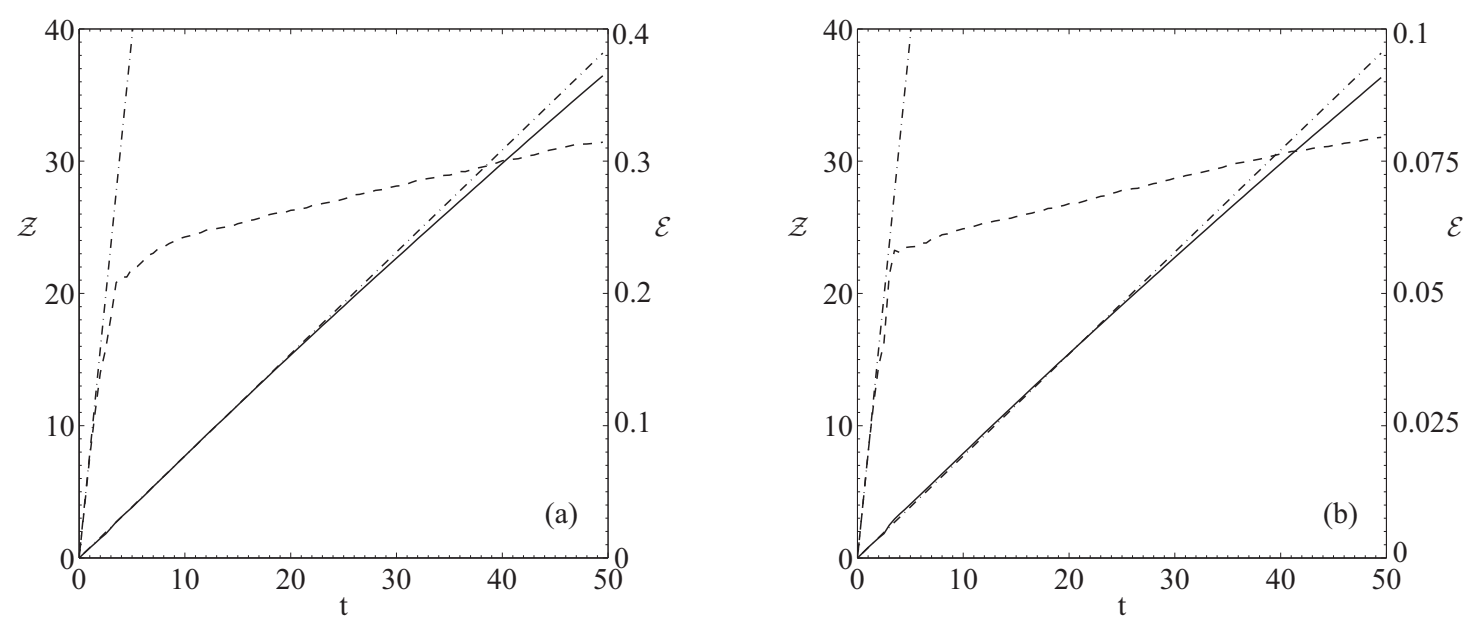

FIG. 2. Temporal evolution of energy (solid) and enstrophy (dashed) for set A (a) and set B (b). The dash-dotted lines correspond to the theoretical growths.
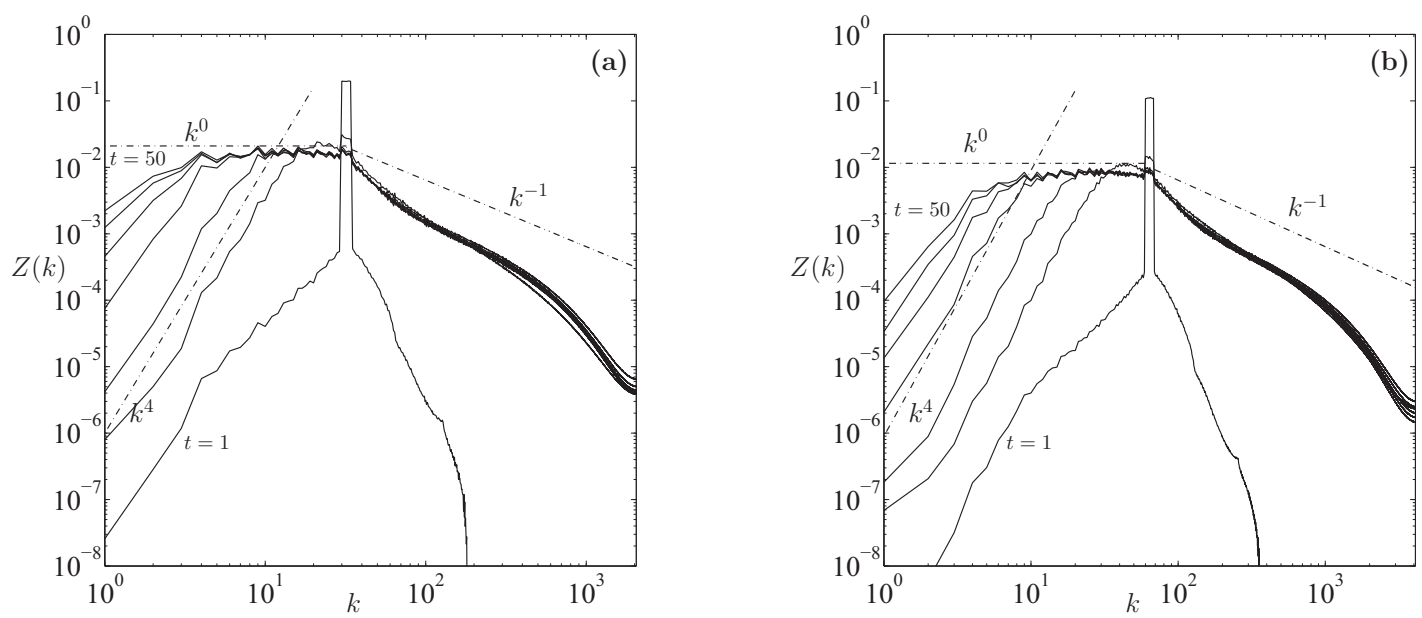

FIG. 3. Enstrophy spectra at increasing times $t=1,5,10,20,30,40$ and 50 (from right to left) for set A (a) and set B (b). Spectra are normalised by total enstrophy.

in wavenumbers larger than $k_{f}$, exhibits an intermediate $k^{-1}$ spectrum, albeit with a steepening just to the right of the forcing, possibly due again to presence of coherent vortices. However, the wavenumber ranges here are too short to make any comparison with theoretical prediction meaningful. For wavenumbers lying in the inverse energy cascade range, i.e. wavenumbers smaller than $k_{f}$, the spectra exhibit a plateau between the forcing wavenumber $k_{f}$ and a smaller wavenumber $k_{b}(t)$ corresponding to the front of the inverse cascade. For $k \ll k_{b}$, the enstrophy drops sharply with a slope which, at intermediate times, is close to 4 , but which decreases as soon as the energy at the domain scale becomes significant, this occurring around $t=20$ for set $\mathrm{A}$ and around $t=30$ for set $B$. In the meantime, $k_{b}$ decreases and the length of the plateau increases. The presence of the plateau, which corresponds to an energy spectrum of $k^{-2}$, is in line with the results of $\operatorname{Scott}^{2}$ and Vallgren ${ }^{20}$ and provides further evidence that Kraichnan's similarity hypotheses is not valid.

The spectra are next fit to a simple analytical form to examine their evolution more precisely. Given the observed shape of the enstrophy spectra, we propose the following analytical form

$$
Z(k)=\left\{\begin{array}{cc}
c\left(k / k_{b}\right)^{4} & 0 \leq k<k_{b} \\
c & k_{b} \leq k<k_{f}
\end{array},\right.
$$

where $k_{b}$ is an unknown time dependent wavenumber corresponding to the front of the enstrophy plateau moving up-scale and $c$ is the constant value of the plateau. This model is limited to wavenumbers smaller than the forcing wavenumber since for $k>k_{f}$ the observed spectra are approximately independent of time. We fit the numerical spectra up to $k_{f}$ by simply integrating the analytical 

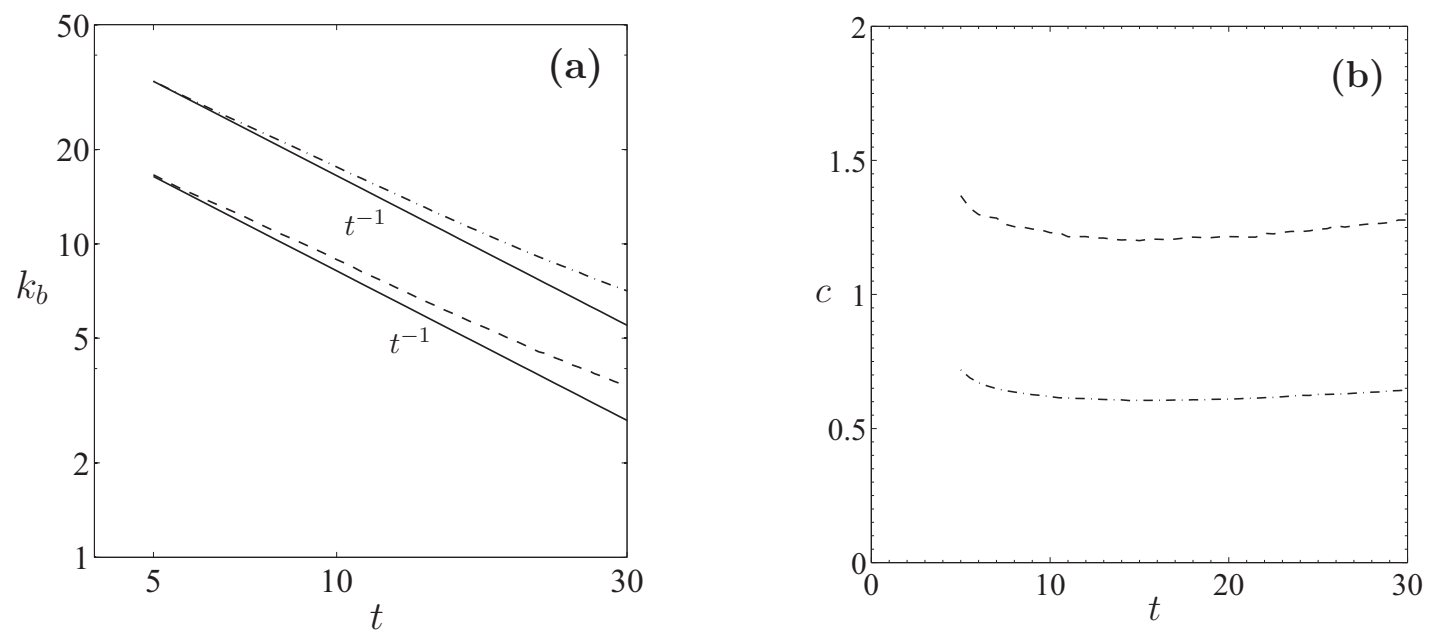

FIG. 4. Time evolution of the coefficients $k_{b}$ (a) and $c$ (b) corresponding to the analytical model 2: the dashed line for set A and the dash-dotted lines for set B. In figure (a) a logarithmic scale is used and the solid lines show the theoretical prediction.

spectrum (2) to obtain the total energy and enstrophy in the range $k<k_{f}$ :

$$
\begin{aligned}
& \mathcal{Z}_{f}=\int_{0}^{k_{f}} Z(k) d k=c\left(k_{f}-\frac{4}{5} k_{b}\right), \\
& \mathcal{E}_{f}=\int_{0}^{k_{f}} Z(k) k^{-2} d k=c\left(\frac{4}{3 k_{b}}-\frac{1}{k_{f}}\right) .
\end{aligned}
$$

The parameters $k_{b}$ and $c$ are then determined by equat$\operatorname{ing} \mathcal{E}_{f}$ and $\mathcal{Z}_{f}$ to the measured energy and enstrophy in the same range.

The temporal evolution of $k_{b}$ and $c$, determined in this way, is shown in figure 4 for times up to $t=30$, corresponding to when the flow evolution begins to be influenced by the finite domain. Note that for constant $c$, the measured linear growth in energy would correspond to $k_{b} \sim t^{-1}$, for $k_{b} \ll k_{f}$; notably, this is a faster growth at large scales than the estimate $t^{-3 / 2}$ predicted by Kraichnan ${ }^{3}$. Figure 4(a) shows that $k_{b}$ does indeed scale close to $t^{-1}$; the slight departure at later times is associated with the slight increase in $c$ as the domain size is approached. At these times, the large-scale part of the spectra that drops sharply for $0 \leq k<k_{b}$ starts to become shallower than $k^{4}$, and the spectral shape is no longer accurately represented by the analytical form 2 .

We emphasize that the values of $k_{b}$ and $c$ are not obtained by any curve fitting to the measured spectrum; rather they are determined uniquely by equating the total measured energy and enstrophy in the region $k<k_{f}$ with the quantities $\mathcal{E}_{f}$ and $\mathcal{Z}_{f}$ from (3). As long as the finite size of the simulation box can be ignored, the analytical form (2) accurately represents the enstrophy spectra for $k<k_{f}$. Both the analytical form and the measured spectrum are shown superposed in figure 5 and can be seen to be in close agreement in this range.

\section{Vortex population characteristics}

We now consider the population of coherent vortices that emerge at the forcing scale and grow through multiple interactions. These are good candidates for the deviation of the present results from the theoretical predictions. Indeed, Kraichnan derived his model assuming that energy transfers occur locally in spectral space through local triad interactions. His model does not take into account the effect of coherent vortices, which are localised in physical space and therefore are widely distributed in spectral space. This was argued previously by Dritschel et al. ${ }^{32}$ in the case of freely-decaying turbulence, for which it was shown that vortices are responsible for the (there strong) deviation between their numerical results and Batchelor's theory. In forced turbulence, Danilov \& Gurarie $^{12}$ and Vallgren ${ }^{20}$ also found that the effect of coherent vortices is significant, and, in particular, that the dominant triad interactions are highly nonlocal. Here we extend their analyses by examining characteristics of the vortex population, in particular their number density and their core vorticity as a function of their size.

We consider a decomposition of the full vorticity field into a component associated with coherent vortices and a residual. To define the coherent part we first identify contiguous regions of vorticity whose magnitude is above the rms value, following the method described in Dritschel et $a l .{ }^{32}$ for the freely decaying case. We then consider the shape of each contiguous region. In the freely decaying case this was done simply by considering the aspect ratio of an ellipse fit to each region. In the forced case, however, vortices are often highly distorted by the forcing field. A more appropriate criterion, we found, is

$$
\delta_{v}=\frac{\left(\iint_{A} \omega d A\right)^{2}}{2 \pi \iint_{A} \omega^{2}\left|\boldsymbol{x}-\boldsymbol{x}_{v}\right|^{2} d A}>0.5
$$



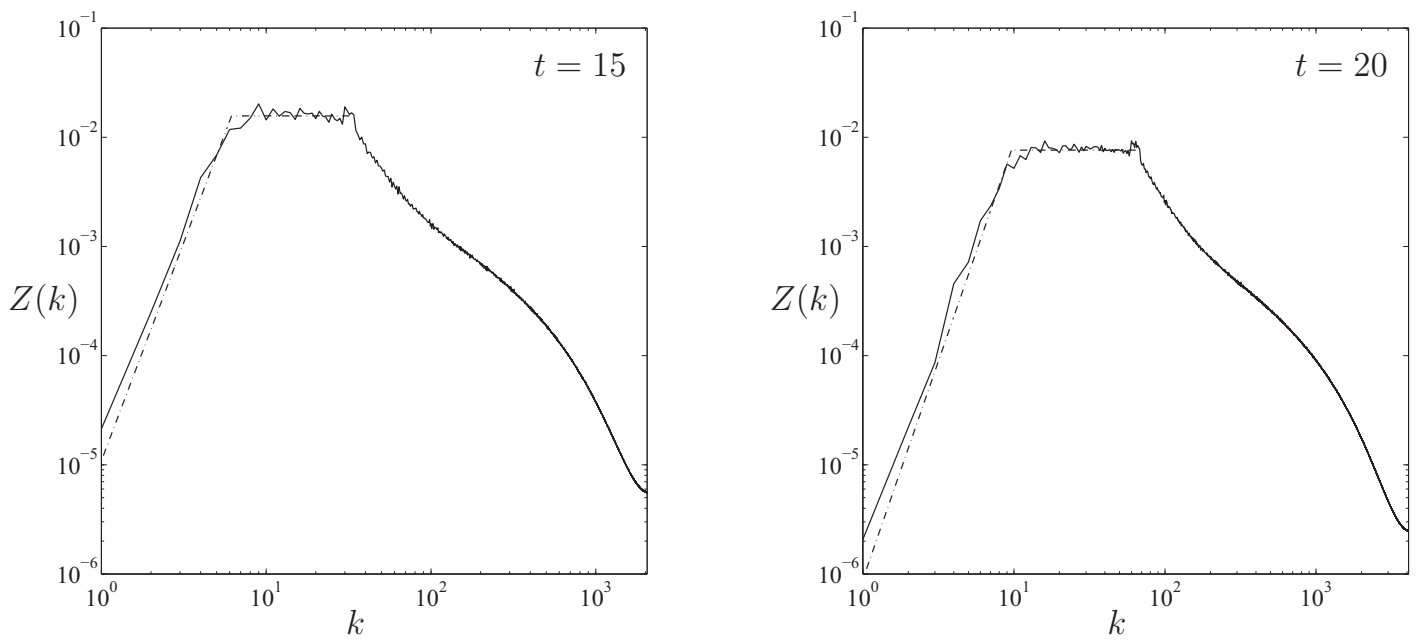

FIG. 5. Superposition of the numerical (solid) and analytical (dashed-dotted) enstrophy spectra for set A at time $t=15$ (left) and set $\mathrm{B}$ at time $t=20$ (right). Spectra are normalised by total enstrophy.
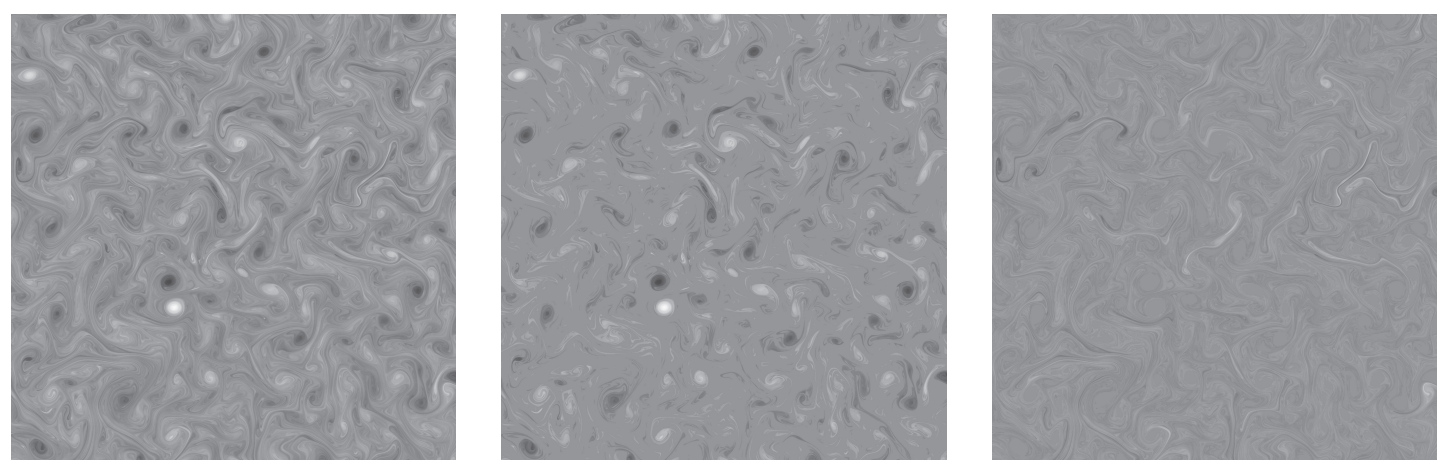

FIG. 6. Decomposition of the vorticity field (left) into coherent (middle) and incoherent part (right). The images are screenshots of one of the simulations in set B at time $t=10$. Only one sixteenth of the domain is shown.

where

$$
\boldsymbol{x}_{v}=\frac{1}{\mathcal{Z}_{v}} \iint_{A} \omega^{2} \boldsymbol{x} d A \text { and } \quad \mathcal{Z}_{v}=\iint_{A} \omega^{2} d A
$$

are the centroid and enstrophy of the region. Note that $0<\delta_{v} \leq 1$ and that $\delta_{v}=1$ corresponds to a circular patch of uniform vorticity. This criterion permits vortices to be more distorted, so long as they have a concentrated centre of high vorticity (as is often found). The integrals in equation 4 are evaluated with the full vorticity field on the ultra-fine re-contouring grid, summing over contiguous regions on this grid. Figure 6 illustrates the vortex identification procedure for a representative simulation in set B; the original vorticity field is shown on the left, the coherent vortices in the middle, and the residual on the right.

With this decomposition, enstrophy spectra associated with each field are obtained, shown in figure 7 for set A at $t=15$ and set $\mathrm{B}$ at $t=20$. As expected, most of the energy and enstrophy is contained within the field of coherent vortices, except at the very smallest scales.
At these times, the population of coherent vortices accounts for $72 \%$ of the total enstrophy. Moreover, we find that the vortex population exhibits a $k^{-2}$ energy spectrum $\left(k^{-2} Z(k)\right)$ in the inverse cascade range while the background residual follows Kraichnan's prediction with a spectrum $\propto k^{-5 / 3}$, indicating a structureless field of filamentary debris, consistent with Figure 6.

We next examine the number density distribution of the vortex population. Benzi et al. ${ }^{33}$ argued that algebraic energy spectra of the form $k^{-p}$ for $p>3$ may be associated with the emergence of a distribution of vortex sizes, i.e. an algebraic number density distribution $n_{v}(A)=c A^{-q}$, provided that the average vorticity $\omega_{v}$ in each vortex does not vary significantly with its area $A$. We can write the coherent enstrophy as

$$
\mathcal{Z}_{\mathrm{coh}}=\int Z_{\mathrm{coh}}(k) d k=\frac{1}{A_{s}} \int \omega_{v}^{2} A n_{v}(A) d A
$$

where $A_{s}$ is the size of the domain considered. Identifying 

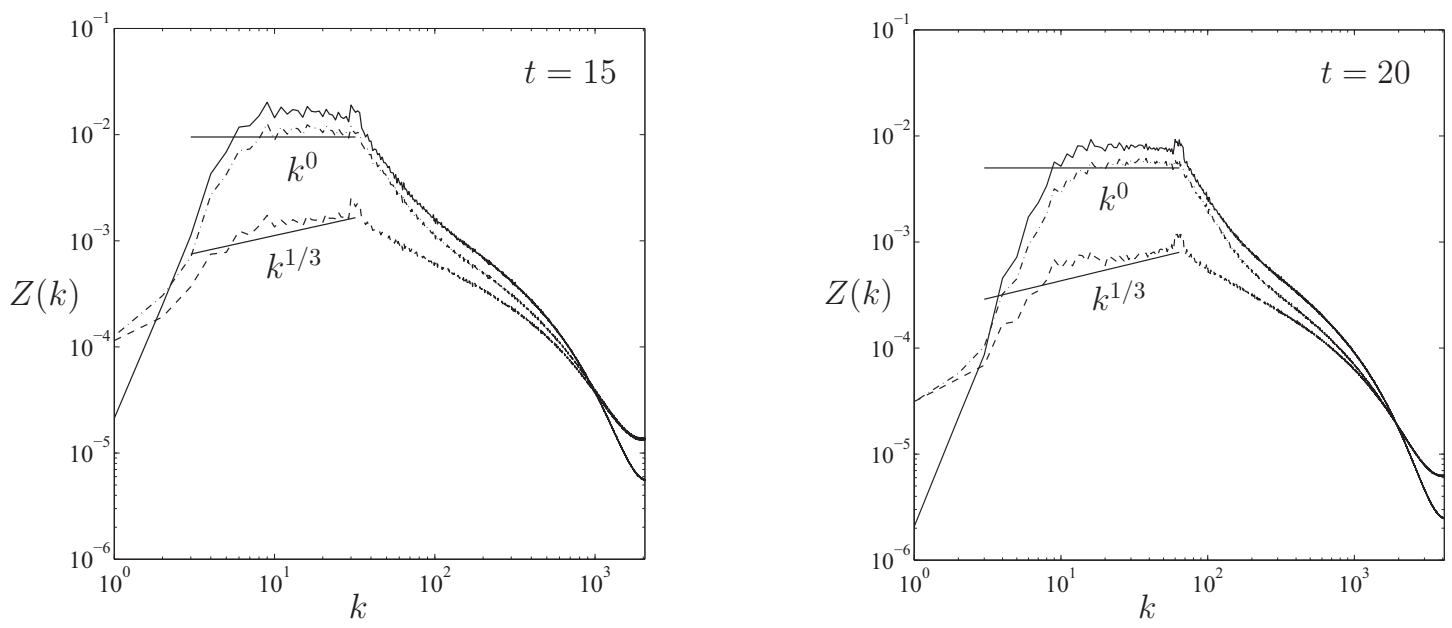

FIG. 7. Decomposition of the enstrophy spectra (solid) into coherent (dash-dotted) and incoherent (dashed) parts. These figures correspond to set $\mathrm{A}$ at $t=15$ (left) and set B at $t=20$ (right). Spectra are normalised by total enstrophy.
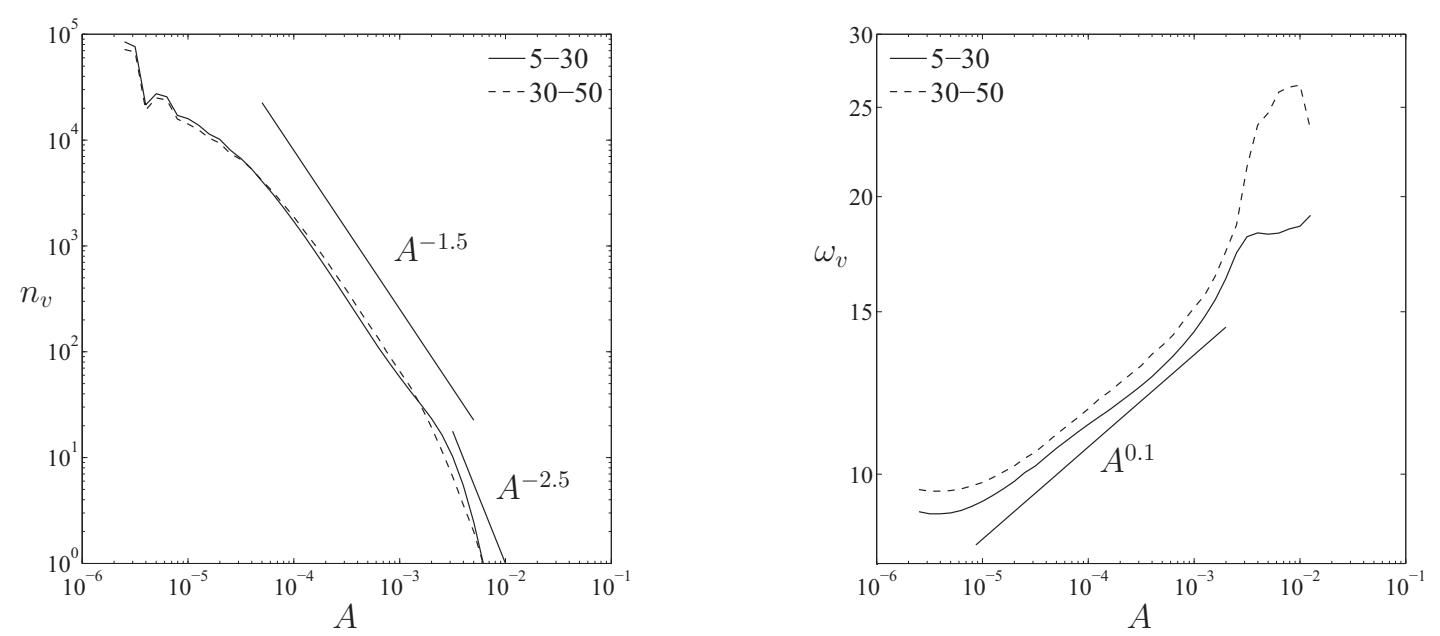

FIG. 8. Vortex population characteristics for set B: vortex number (left) and average vorticity (right) distribution function of vortex area.

$A$ with $k^{-2}$, it follows that

$$
Z_{\mathrm{coh}}(k) \propto k^{-5+2 q} .
$$

Figure 8 shows the characteristics of the vortex population for set $\mathrm{B}$, averaged over two periods : from $t=5$ to $t=30$ and from $t=30$ to $t=50$. Considering the vortex number density distribution, one can identify a change in the slope occurring at an area that corresponds to the scale of forcing, i.e. $A_{f} \sim k_{f}^{-2}$. For vortices having an area smaller than $A_{f}$, the number density distribution scales like $A^{-1.5}$ over two decades. These trends are not significantly modified by the finite size effects from $t=30$ as curves superimpose. The average vorticity $\omega_{v}$ shown in the lower panel grows with vortex area, though varies by less than a factor of about 3 from the smallest to the largest areas.
By (7), the number density distribution $n_{v}(A) \propto A^{-1.5}$ would correspond to the (coherent) enstrophy spectrum $Z_{\text {coh }} \propto k^{-2}$ in the enstrophy inertial range, a form consistent with a prediction of Saffman ${ }^{34}$ who considered the effect of discontinuities in the vorticity field. For big vortices having an area larger than $A_{f}$, the distribution seems to scale like $A^{-2.5}$, consistent with the $k^{0}$ slope observed in the energy inertial range. However, because the separation between the forcing scale and the box size is relatively limited here, the range of areas is too small to draw any definite conclusion. It is noteworthy that the size of the largest vortex appears to be significantly smaller than would be expected on the basis of $k_{b}$. Moreover, the systematic growth in the average vorticity with vortex area observed in figure 8, especially at large areas, puts in question the simple relation between number 
density $n_{v}(A)$ and enstrophy spectrum $Z_{\text {coh }}(k)$ argued by Benzi et al. ${ }^{33}$ for a dilute population of vortices having comparable vorticity.

\section{CONCLUSIONS}

We have examined novel high-resolution numerical simulations of forced two-dimensional turbulence to investigate further the validity of Kraichnan's classical theory ${ }^{3}$. Our approach differs from previous numerical studies in three key respects. First, we have made use of a powerful hybrid numerical algorithm $(\mathrm{CLAM})^{1}$, particularly suited to the simulation of geophysical flows and two-dimensional turbulence, and previously demonstrated to be much more efficient than standard pseudo spectral methods commonly used ${ }^{28}$. Second, no largescale dissipation has been used to avoid any bias in the energy cascade ${ }^{12,20,21}$ : in place of temporal averaging, ensemble averages have been used to provide accurate statistics, consistent with Kraichnan's theory. Third, the ratio $k_{\max } / k_{f}$ between the maximum resolved wavenumber and the forcing wavenumber is sufficiently large (here 64 ), to give room for the emergence of a vortex population which, in time, dominates energetically, enables a strong inverse energy cascade to develop, and steepens the large-scale energy spectrum via highly-nonlocal interactions. The lack of large-scale dissipation means that our simulations are not stationary in the strict sense, because energy grows continuously, but may be considered quasi-stationary in the sense that the energy plateau remains at a constant level, with only the front advancing to the left in time. The energy distribution in a subrange of the inverse cascade is thus stationary. In fact, it is precisely this quasi-stationary situation that was originally considered by Kraichnan, in which energy cascades undissipated towards ever large scales.

The results obtained in the present work confirm the results of Scott and the more recent results of Vallgren ${ }^{20}$ : in the energy inertial range the observed enstrophy spectrum is $Z(k) \propto k^{0}$, rather than the $k^{1 / 3}$ spectrum predicted by Kraichnan. This $k^{0}$ spectrum is associated with a transfer of energy to large scales that scales like $t^{-1}$, in contrast to the $t^{-3 / 2}$ prediction of Kraichnan. We propose a simple analytical form for the enstrophy spectrum at scales larger than the forcing scale that matches closely the numerically obtained spectrum.

The deviation from Kraichnan's scalings is associated with the emergence of a population of vortices that violates Kraichnan's assumption of local triad interactions. Kraichnan's scalings can of course be obtained when the forcing is closer to the truncation scale than here, or when a linear drag is used to reach a stationary state, as both of these effects act to suppress vortex formation at the forcing scale. When these effects are absent, however, the formation of vortices at the forcing scale leads to a distortion of the inverse cascade and the above steepening of the energy spectrum. To demonstrate the role of coherent vortices, a vortex identification criterion was used to separate the full vorticity field into coherent and residual parts. The coherent part was shown to contain about $90 \%$ of the total energy of the flow and to exhibit a $k^{0}$ enstrophy spectrum in the energy inertial range. The spectrum associated with the residual part follows Kraichnan's $k^{1 / 3}$ prediction.

It could be argued whether the steepening of the inverse cascade observed here is due simply to an insufficient length of the inertial range, which in the experiments discussed above spans slightly less than one decade in wavenumber space. By analogy, in studies of the direct enstrophy cascade, energy spectra steeper than $k^{-3}$ may be obtained when the high wavenumber inertial range is short and vortices that form near the forcing scale dominate the spectrum; as the inertial range is increased the influence of the vortices diminishes and the $k^{-3}$ spectrum is recovered. However, in this regard, a fundamental asymmetry between the role of coherent vortices in the direct and inverse cascades should be noted, namely, that due to the tendency of vortices to merge and grow in size they have a more persistent effect on the inverse cascade than on the direct cascade. Vortices created at the forcing scale eventually populate a range of scales larger than the forcing scale, populating the inverse cascade. In contrast to the case of the direct cascade, therefore, it appears less likely that simply increasing the length of the inverse cascade will diminish the influence of coherent vortices and recover the Kraichnan scaling.

To emphasize the distribution of vortices across scales, the distribution of enstrophy in spectral space was also related to the number density distribution of coherent vortices in physical space, following arguments due to Benzi et al. ${ }^{33}$. The range in vortex area is too small to be conclusive but the scaling of the vortex number density distribution appears to be close to $n_{v}(A) \propto A^{-2.5}$, consistent with the scaling of $k^{0}$ for the enstrophy in spectral space. Extending the range of vortex area would require a significant increase in both resolution and forcing wavenumber, unfortunately well beyond the capabilities of current computers.

\section{ACKNOWLEDGMENTS}

Jérôme Fontane is supported by the European Community in the framework of the CONVECT project under Grant number PIEF-GA-2008-221003.

${ }^{1}$ D. Dritschel and J. Fontane, "The combined Lagrangian advection method," J. Comp. Phys., 229, 5408-5417 (2010).

${ }^{2} \mathrm{R}$. Scott, "Nonrobustness of the two-dimensional turbulent inverse cascade," Phys. Rev. E, 75, 046301 (2007).

${ }^{3}$ R. Kraichnan, "Inertial ranges in two-dimensional turbulence," Phys. Fluids, 10, 1417-1423 (1967).

${ }^{4} \mathrm{G}$. Batchelor, "Computation of the energy spectrum in homogeneous two-dimensional turbulence," Phys. Fluids, 12, II-233-II239 (1969).

${ }^{5}$ R. Kraichnan, "Inertial-range transfer in two- and threedimensional turbulence," J. Fluid Mech., 47, 513-524 (1971). 
${ }^{6}$ U. Frisch and P. Sulem, "Numerical simulation of the inverse cascade in two-dimensional turbulence," Phys. Fluids, 27, 19211923 (1984).

${ }^{7}$ M. Maltrud and G. Vallis, "Energy and enstrophy transfer in numerical simulations of two-dimensional turbulence," Phys. Fluids, 5, 1760-1775 (1993).

${ }^{8} \mathrm{~L}$. Smith and V. Yakhot, "Bose condensation and small-scale structure generation in a random force driven 2D turbulence," Phys. Rev. lett., 71, 352-355 (1993).

${ }^{9}$ L. Smith and V. Yakhot, "Finite-size effect in forced twodimensional turbulence," J. Fluid Mech., 274, 115-138 (1994).

${ }^{10} \mathrm{~V}$. Borue, "Spectral exponent of enstrophy cascade in stationary two-dimensional homogeneous turbulence," Phys. Rev. Lett., 71, 3967-3970 (1993)

${ }^{11} \mathrm{~V}$. Borue, "Inverse energy cascade instationary two-dimensional homogeneous turbulence," Phys. Rev. Lett., 72, 1475-1478 (1994).

${ }^{12}$ S. Danilov and D. Gurarie, "Forced two-dimensional turbulence in spectral and physical space," Phys. Rev. E, 63, 061208 (2001).

${ }^{13}$ C. Pasquero and G. Falkovich, "Stationary spectrum of vorticity cascade in two-dimensional turbulence," Phys. Rev. E, 65, 056305 (2002).

${ }^{14}$ S. Chen, R. Ecke, G. Eyink, X. Wang, and Z. Xiao, "Physical mechanism of the two-dimensional cascade," Phys. Rev. Lett., 91, 214501 (2003).

${ }^{15}$ J. Paret and P. Tabeling, "Experimental observation of the twodimensional inverse energy cascade," Phys. Rev. Lett., 79, 41624165 (1997).

${ }^{16}$ J. Paret and P. Tabeling, "Intermitency in the two-dimensional inverse cascade of energy: exeprimental observations," Phys. Fluids, 10, 3126-3136 (1998).

${ }^{17} \mathrm{M}$. Rutgers, "Forced 2D turbulence: experimental evidence of simultaneous inverse energy and forward enstrophy cascades," Phys. Rev. Lett., 81, 2244-2247 (1998).

${ }^{18}$ J. Paret, M. Jullien, and P. Tabeling, "Vorticity statistics in the two-dimensional entrophy cascade," Phys. Rev. Lett., 83, 34183421 (1999).

${ }^{19} \mathrm{C}$. Tran and J. Bowman, "Robustness of the inverse cascade in two-dimensional turbulence," Phys. Rev. E, 69, 036303 (2004).

${ }^{20} \mathrm{~A}$. Vallgren, "Infrared number dependency of the twodimensional inverse energy cascade," J. Fluid Mech., 667, 463473 (2011).

${ }^{21}$ S. Sukoriansky, B. Galperin, and A. Chekhlov, "Large scale drag representation in simulations of two-dimensional turbulence," Phys. Fluids, 11, 3043-3053 (1999).

${ }^{22} \mathrm{G}$. Boffetta, "Energy and enstrophy fluxes in the double cascade of two-dimensional turbulence," J. Fluid Mech., 589, 256-260 (2007).
${ }^{23} \mathrm{G}$. Boffetta and S. Musacchio, "Evidence for the double cascade scenario in two-dimensional turbulence," Phys. Rev. E, 82, 016307 (2010).

${ }^{35} \mathrm{In}$ addition to the cases involving frictional dissipation, Boffetta \& Musacchio considered a single high resolution calculation with no friction in the quasi-stationary regime. The results of that calculation are difficult to discern (see their figure 2) but careful examination again suggests an energy spectrum closer to $k^{-2}$, consistent with the present paper; this conclusion was not drawn in that paper.

${ }^{25}$ D. Dritschel, "Contour surgery: a topological reconnection scheme for extended integrations using contour dynamics," J. Comput. Phys., 77, 240-266 (1988).

${ }^{26}$ D. Dritschel, "Contour dynamics and contour surgery: numerical algorithms for extended, high-resolution modelling of vortex dynamics in two-dimensional, inviscid, incompressible flows," Computer Phys. Rep., 10, 77-146 (1989).

${ }^{27} \mathrm{D}$. Dritschel and R. Scott, "On the simulation of nearly inviscid two-dimensional turbulence," J. Comp. Phys., 228, 2707-2711 (2009).

${ }^{28}$ J. Fontane and D. Dritschel, "The HyperCASL algorithm: a new approach to the numerical simulation of geophysical flows," J. Comp. Phys., 228, 6411-6425 (2009).

${ }^{29}$ D. Dritschel and M. Ambaum, "A contour-advective semiLagrangian numerical algorithm for simulating fine-scale conservative dynamical fields," Quart. J. Roy. Meteorol. Soc., 123, 1097-1130 (1997).

${ }^{30}$ D. Dritschel and M. Ambaum, "The diabatic contour advective semi-Lagrangian algorithm," Mon. Weather Rev., 134, 25032514 (2006).

${ }^{31}$ D. Dritschel and J. Fontane, "The HyperCASL algorithm," in $I U$ TAM symposium on Turbulence in the Atmosphere and Oceans, edited by Springer (2010).

${ }^{32}$ D. Dritschel, R. Scott, C. Macaskill, G. Gottwald, and C. Tran, "A unifying scaling theory for vortex dynamics in twodimensional turbulence," Phys. Rev. Lett., 101, 094501 (2008).

${ }^{33}$ R. Benzi, M. Colella, M. Briscolini, and P. Santangelo, "A simple point vortex model for two-dimensional decaying turbulence," Phys. Fluids A, 4, 1036-1039 (1992).

${ }^{34} \mathrm{P}$. Saffman, "On the spectrum and decay of random twodimensional vorticity distributions at large Reynolds number," Stud. Appl. Math., 50, 377-383 (1971).

${ }^{35} \mathrm{In}$ addition to the cases involving frictional dissipation, Boffetta \& Musacchio considered a single high resolution calculation with no friction in the quasi-stationary regime. The results of that calculation are difficult to discern (see their figure 2) but careful examination again suggests an energy spectrum closer to $k^{-2}$, consistent with the present paper; this conclusion was not drawn in that paper.. 